\title{
Competência Social e Autismo: O Papel do Contexto da Brincadeira com Pares ${ }^{1}$
}

\author{
Cláudia Sanini \\ Maúcha Sifuentes \\ Cleonice Alves Bosa ${ }^{2}$ \\ Universidade Federal do Rio Grande do Sul
}

\begin{abstract}
RESUMO - Observa-se o esforço dos pesquisadores em delinear e avaliar intervenções para facilitar o desenvolvimento da interação social em crianças com autismo e seus pares, em situações de inclusão escolar. Entre os resultados controversos dos estudos estão os que se referem ao papel do contexto das brincadeiras, isto é, se livre ou dirigida, na promoção da competência social dessas crianças. O objetivo deste estudo foi revisar criticamente a literatura sobre o tema, buscando-se evidências sobre que tipo de contexto de brincadeira tende a promover as interações entre pares, examinando-se as questões metodológicas que cercam esse debate. A conclusão foi de que ambos os contextos promovem o desenvolvimento da competência social, mas o livre tende a ser mais duradouro e espontâneo.
\end{abstract}

Palavras-chave: autismo, interação social, inclusão escolar, brincadeira livre, brincadeira dirigida

\section{Social Competence and Autism: The Role of the Context of Play with Peers}

\begin{abstract}
There is a substantial effort of researchers in designing and assessing interventions aiming to promote the social interaction between children with autism and their peers, in the context of inclusion. Among the results that are controversial are the issues related to the role of the play context (free or directed) in the promotion of children's social competence. The aim of this study was to critically review the literature about this topic by searching evidences about what sort of play context tend to promote peer interaction and by examining the methodological issues that surround this debate. The conclusion is that both contexts promote the development of social competence, but the free one tends to be more permanent and spontaneous.
\end{abstract}

Keywords: autism, social interaction, school inclusion, free play, structured play

Os fatores que favorecem ou, ao contrário, impedem a interação social de crianças com autismo e seus pares têm sido um foco crescente de interesse de pesquisadores na área da psicologia do desenvolvimento. Umas das questões que se apresenta controversa é acerca do tipo de contexto de brincadeira que mais promoveria essas interações: se aquele definido como livre, isto é, no qual as interações ocorrem de forma espontânea, ainda que encorajadas pelos educadores, ou o dirigido. Neste último, em geral, há instruções prévias sobre como os sujeitos devem se comportar, em situações igualmente previamente estruturadas. Neste estudo pretende-se examinar algumas pesquisas que têm se dedicado a essa investigação, colocando-se em relevo especialmente os aspectos metodológicos que as nortearam. Parte-se de uma breve definição de autismo e do construto de competência social, no qual as discussões sobre interação social se ancoram, antes da apresentação dos estudos propriamente ditos. Busca-se, com isto, contribuir para as práticas pedagógicas cujo foco é o desenvolvimento social da criança com autismo, em situação de inclusão escolar.

1 Nossos agradecimentos à Prof. ${ }^{a}$ Dr. ${ }^{a}$ Ana Almeida, da Universidade do Minho, Portugal, pelas suas contribuições.

2 Endereço para correspondência: Instituto de Psicologia, UFRGS, Rua Ramiro Barcelos 2600, Santa Cecília, Porto Alegre, RS. CEP 90035003. E-mail: cleobosa@uol.com.br

\begin{abstract}
Autismo
O autismo é uma condição que afeta as principais áreas do desenvolvimento, quais sejam, a interação social e a linguagem, além da ocorrência de comportamentos repetitivos e estereotipados (Associação Psiquiátrica Americana, 2002). Esses últimos se expressam de diferentes formas: na brincadeira, que pode carecer de criatividade e espontaneidade; na fala, que pode ser ecolálica; e no desenvolvimento motor, caracterizado por repetições involuntárias e sem aparente função (ex: rituais e maneirismos) (Bosa, 2002). As manifestações dessas dificuldades poderão variar de acordo com o nível desenvolvimental e a idade cronológica da criança, entre outros fatores (APA, 2002). Quanto maior o comprometimento cognitivo, maior a tendência a se isolar e a não se comunicar (Klin, 2006). Por outro lado, a ideia de que a criança com autismo não demonstra afeto e é inquestionavelmente distante e não-comunicativa, também não tem recebido apoio empírico (Bosa, 2002). Há fartas evidências sobre a capacidade da criança com autismo em desenvolver relações de apego a seus cuidadores e de responder à interação social (ver Sanini, Ferreira, Souza, \& Bosa, 2008). Os comprometimentos que parecem ser substanciais são, de fato, os da habilidade de atenção compartilhada, especialmente a iniciativa para buscar e manter a interação, de forma recíproca e espontânea.
\end{abstract}


De acordo com Bosa (2002), o comportamento social de crianças com autismo, mais do que um isolamento "proposital" parece decorrer, principalmente, do comprometimento na ausência de compreensão acerca do que se quer dela. Em outras palavras, da dificuldade em compreender intenções e outros estados mentais, como crenças, pensamentos ou sentimentos das demais pessoas, a tal ponto que o autismo é conhecido como uma "cegueira mental" (Baron-Cohen \& Bolton, 1993). Essa capacidade para compreender, explicar e predizer o comportamento humano em termos de estados mentais, tão usual nas interações sociais, tem sido convencionalmente chamada de Teoria da Mente (Premack \& Woodruff, 1978), um construto nascido da psicologia cognitiva e que se caracteriza por um sistema de inferências que permite fazer previsões (teorizações) sobre o comportamento dos outros. Como tais estados mentais não são diretamente observáveis, essa habilidade é vista como uma "teoria". Devido a esse comprometimento, o estímulo natural da interação social a essas crianças, oportunizado, por exemplo, pela inclusão escolar, o mais cedo possível, possibilita a ampliação do repertório de habilidades sociais e de aceitação pelos pares. Desse modo, o isolamento constante pode ser evitado, especialmente nos anos pré-escolares.

\section{Competência social e autismo}

Competência social é definida como um conjunto de comportamentos aprendidos no decorrer das interações sociais, especialmente, nas interações com pares. Assim, o desenvolvimento da competência social, em uma perspectiva organizacional-relacional (Waters \& Sroufe, 1983) procura ressaltar a variedade de soluções adaptativas, empregadas em diferentes contextos e situações, que permitem à criança desenvolver-se socialmente. $\mathrm{O}$ foco são os recursos que a criança utiliza para lidar com esse desafio, não se tratando apenas de "aptidões". Isso significa que, nos casos de crianças com autismo, até mesmo seus sintomas podem constituir recursos que auxiliam a interação. Por exemplo, as estereotipias motoras de uma criança podem ser vistas, pelos colegas, como "habilidades" e despertar admiração, mais do que rechaço.

$\mathrm{Na}$ área da psicologia do desenvolvimento, a competência social é uma habilidade que emerge da experiência proporcionada por relações mais próximas e íntimas (Hartup 1989) e constitui a base do desenvolvimento global da criança (cognitivo, emocional, linguagem, etc.). Um dos obstáculos na compreensão do conceito está no uso indistinto entre diversos termos do campo do desenvolvimento social, como, por exemplo, "comportamentos pró-sociais", "condutas socialmente adaptadas", "competência cognitiva" e "competência sociocognitiva", trazendo imprecisão ao conceito (Pizzinato \& Sarriera, 2003). Em especial, é importante ressaltar que o termo competência social não é sinônimo de habilidade social, conquanto sejam conceitos intimamente relacionados. A habilidade social relaciona-se ao desempenho do indivíduo como um todo frente às exigências de uma situação (Del Prette \& Del Prette, 2005). A competência social relaciona-se ao julgamento sobre a qualidade do desempenho individual em determinada circunstância. Assim, é importante considerar a competência social em uma dimensão de desenvolvimento, contextualizada no seu espaço sociocultural (Almeida, 1997).

Hartup (1989) sugere dois tipos de relacionamento que toda criança precisa vivenciar: o vertical e o horizontal. $\mathrm{O}$ relacionamento vertical ocorre entre a criança e alguém com maior poder social e conhecimento (pais, irmãos mais velhos, professores), proporcionando, assim, segurança e proteção. O horizontal caracteriza-se pela igualdade e reciprocidade, uma vez que ocorre entre companheiros da mesma idade e, por isso, permite o desenvolvimento de aspectos sociais, somente experienciados nessa relação, como a cooperação, a competição e a intimidade.

A premissa básica das ideias de Hartup é a de que a competência social é, em sua maior parte, aprendida com os companheiros. As interações com pares fornecem as experiências necessárias para o desenvolvimento de aptidões sociocognitivas, que são a base fundamental para o autoconhecimento e a aprendizagem em geral (Almeida, 1997). Assim, verifica-se que no processo de socialização de crianças e adolescentes, os pais, apesar de atuarem mais precocemente como agentes socializadores sobre a criança, não são os únicos protagonistas no processo de desenvolvimento da personalidade de seus filhos. A importância da interação social reside na noção de que essa habilidade é a base do desenvolvimento infantil e, por conseguinte, está implicada nos processos de desenvolvimento da linguagem e da aprendizagem (Almeida, 1997). A capacidade simbólica é a habilidade que liga todos esses processos e tem sua origem nas experiências de interação entre a criança e seus cuidadores, principalmente durante os "jogos sociais" (Bruner, 1997).

Do ponto de vista cognitivo, da mesma forma, quando uma criança interage com um colega cognitivamente mais "habilidoso", ela pode demonstrar habilidades superiores do que poderia fazê-lo, se estivesse sozinha (Rogers, 2000). Processo similar pode ocorrer na esfera social. Uma criança que interage com um colega socialmente "habilidoso" provavelmente terá uma relação de melhor qualidade - e, então com maior competência - do que a mesma criança interagindo com um colega com menos habilidades nessa área (Rogers, 2000). Nesse caso, crianças com autismo, um transtorno que tem como uma de suas principais características justamente uma dificuldade na interação social, podem ser duplamente beneficiadas com essas experiências. Da mesma forma, crianças com desenvolvimento típico (DT) também se beneficiam dessa relação, na medida em que têm a possibilidade de conviver e aprender com as diferenças. Acredita-se que as consequências dessa experiência para as crianças com DT vão muito além da redução de preconceitos. A consciência e o respeito pelo diferente não somente são instâncias do desenvolvimento moral (Biaggio, 1976), mas também do cognitivo. Isso porque a diversidade, em contraposição ao "padrão", amplia a capacidade simbólica, uma vez que essa experiência transforma a realidade, possibilitando diferentes parâmetros que a criança experimenta sobre o que significa "ser criança" (ex: diferenças no comportamento, nas expectativas, etc.).

Nesse sentido, a escola constitui um espaço essencialmente interativo e reconhecidamente relevante para o desenvolvimento interpessoal e, por isso, é um espaço natural para a relação entre pares. Para Del Prette e Del Prette (2005), o 
investimento da escola na promoção de habilidades sociais pode ser defendido com base em três argumentos: a) a função social da escola, b) as evidências de relação entre habilidades sociais e desempenho acadêmico, e c) as políticas de inclusão.

\section{Inclusão escolar: como promover a relação entre os pares?}

A proposta de inclusão, resultado das recentes mudanças no pensamento da humanidade, demonstra uma preocupação cada vez maior com o ser humano em si, razão pela qual se destaca a atenção especial prestada aos direitos humanos. Deve-se lembrar que a igualdade está relacionada aos direitos humanos e não às características das pessoas, que inerentemente são diferentes e devem ser compreendidas (Baptista, 2006).

No entanto, é lamentável que a formalização dos direitos humanos ocorra mais por meio da criação de leis e menos com base em uma consciência social. Há ainda uma parcela da população que sofre as consequências da exclusão como, por exemplo, as pessoas com deficiências - intelectuais, físicas, múltiplas e/ou sensoriais - e de transtornos do desenvolvimento. Muito já tem sido feito para mudar esse paradigma, o que representa uma visão diferenciada sobre o tema. Porém, muito ainda resta a fazer, já que as questões relacionadas aos benefícios da inclusão ainda têm sido motivo de muita discussão, que envolve desde o desconhecimento de sua proposta até o descrédito sobre a sua real efetividade (Silveira \& Neves, 2006). Por outro lado, é possível verificar consenso em relação a um aspecto: a educação inclusiva é uma proposta viável, mas somente poderá ser efetivada se ocorrerem profundas transformações na política social e no sistema de ensino vigente (Silveira \& Neves, 2006).

Em relação às interações sociais das crianças com necessidades educacionais especiais (NEE) e seus pares com desenvolvimento típico (DT), em situação de inclusão escolar, as pesquisas apontam para aspectos práticos que precisam ser considerados a fim de que a inclusão possa ser real e efetiva. Dentre eles, destaca-se a necessidade de que o processo inclusivo seja realizado o mais cedo possível na vida de uma criança (Fuller \& Jill, 2006), especialmente no caso de crianças com autismo, cuja característica central, como já mencionado, é uma dificuldade na habilidade para formar e manter relações com pares (Associação Psiquiátrica Americana, 2002).

Para Macintosh e Dissanayake (2006), a escola, como um espaço que oportuniza a convivência com pares, associada às características da própria criança, às práticas parentais e à história de interação familiar, completa o quadro das influências mais significativas sobre o comportamento infantil e contribui de diversos modos para a formação do indivíduo por meio do desenvolvimento de comportamentos, habilidades e valores.

De acordo com Batista e Enumo (2004), a carência de oportunidades das crianças com NEE para se relacionarem com outras, impede o refinamento, a experiência e a expansão dos repertórios sociais, reduzindo, assim, a probabilidade para desenvolverem amizades. Consequentemente, essas crianças demonstrarão certa inadequação em seus compor- tamentos, o que leva a uma menor aceitação por parte dos colegas, reforçando, assim, as diferenças existentes.

Apesar da importância do tema, o que se tem observado na literatura é uma escassez de estudos, especialmente nacionais, na área da interação social de crianças com autismo, em situação de inclusão escolar. A carência se torna ainda maior quando o foco são as interações naturais dessas crianças (Anderson, Moore, Godfrey, \& Fletcher-Flinn, 2004; Jahr, Eikeseth, Eldevik, \& Aase, 2007; Macintosh \& Dissanayake, 2006). Na literatura internacional, a predominância recai no uso de metodologias com delineamentos transversais, com ênfase na utilização de programas de intervenção, com brincadeiras dirigidas, isto é, nas quais ocorrem instruções a seus participantes.

\section{Inclusão, Interação Social em Contexto de Brincadeiras Livres ou Dirigidas}

Pesquisadores têm devotado substancial esforço para descrever o curso desenvolvimental das interações sociais entre crianças com autismo, bem como para delinear e avaliar intervenções com fins de facilitar o desenvolvimento dessas competências (McConnell, 2002). Alguns dos resultados nessa área demonstram avanços na competência social dessas crianças, em termos de ganhos no que se refere à capacidade de sustentar a interação social com pares e de reduzir o isolamento e a rigidez comportamental (LeGoff, 2004). Há evidências, também, no sentido de aumentar as iniciativas para brincar, o tempo de envolvimento das crianças com grupos, assim como o nível de sofisticação das brincadeiras, em contexto de inclusão (Nelson, Nelson, McDonnell, Johnston, \& Crompton, 2007).

Uma das estratégias utilizadas tem sido a integração de pares com DT e autismo, no contexto de atividades que envolvam jogos e/ou brincadeiras na escola. Esses pares são treinados, estimulados e reforçados pelos adultos a aumentar as iniciações sociais e também para servirem de modelo para as crianças com autismo (Gonzalez-Lopez \& Kamps, 1997; Kamps et al., 1992; Kok, Kong, \& Bernard-Opitz, 2002; Terpstra \& Tamura, 2007; Whitaker, 2004; Yang, Wolfberg, Wu, \& Hwu, 2003).

Esses estudos que utilizam estratégias de intervenção mostram, em sua maioria, resultados positivos, o que têm estimulado a realização de investigações nessa área. No estudo de Kamps et al., (1992), por exemplo, foram formados grupos com a finalidade de trabalhar as habilidades sociais e, assim, facilitar o aumento das interações entre alunos com autismo e seus pares com DT (os quais receberam treinamento previamente). O estudo ocorreu em uma classe integrada do primeiro ano do Ensino Fundamental. Participaram três crianças-foco de 7 anos de idade, com autismo de "alto funcionamento", isto é, sem deficiência mental associada. A avaliação dos dados foi realizada por dois codificadores independentes. A média dos níveis de concordância entre os codificadores foi relativamente alta. $\mathrm{Na}$ análise de frequência de interações sociais, os percentuais de concordância variaram entre $33 \%$ e $100 \%$, enquanto na análise do tempo engajado em interações sociais, de $25 \%$ a $100 \%$. A intervenção foi avaliada a partir de um delineamento de linha 
de base múltipla. Os resultados evidenciaram um aumento na frequência de engajamento e de duração das interações sociais, bem como na responsividade dos alunos com autismo e dos pares entre si. Em especial, destaca-se que os achados demonstraram também um aumento no número de iniciativas sociais das crianças com autismo, uma vez que esse aspecto é o mais comprometido nessas crianças, em detrimento às respostas sociais. Contudo, não foi avaliada a efetividade desse tipo de intervenção em casos mais severos de autismo.

Da mesma forma, na pesquisa de Gonzalez-Lopez e Kamps (1997), crianças foram treinadas em habilidades sociais por professores da educação especial em uma variedade de situações (ex.: cumprimentar, seguir instruções, pedir ajuda, etc.). As crianças com DT receberam informações acerca do autismo e treinamento sobre os procedimentos para gerenciar comportamentos básicos (ex: isolamento, estereotipias, etc.). Participaram quatro crianças com autismo e 12 crianças com DT, com idade entre 5 e 7 anos. Foram realizadas sessões em grupos, formados por uma criança com autismo e três com DT. Os resultados mostraram um aumento na frequência e duração das interações de todas as crianças envolvidas. Ou seja, nas crianças com autismo, a frequência média das interações aumentou quando foi introduzido o treinamento das habilidades sociais e do reforço positivo das interações.

Em um outro estudo (Kok et al., 2002) foram comparados os efeitos da brincadeira dirigida (maior direcionamento de um experimentador) - e da facilitada (método de ensino casual) na promoção da espontaneidade e responsividade na comunicação e no comportamento durante as brincadeiras, em crianças com autismo. As crianças estavam na faixa etária de 4 e 5 anos, em uma escola de Cingapura. Foi empregado um delineamento de linha de base múltipla. A sequência da instrução e aplicação dos métodos foi contrabalanceada. As crianças com DT foram solicitadas a interagir com as crianças com autismo nas duas situações. Uma análise qualitativa mostrou uma avaliação positiva por parte das crianças com DT acerca dessa experiência. Além disso, os relatos dos cuidadores das crianças com autismo validaram os ganhos clínicos observados, pois todos perceberam "mudanças que variaram de pequenas a notáveis" na área da comunicação, sendo que a maioria relatou pequenas mudanças na área do jogo e uma diminuição nos problemas comportamentais. Também foi relatado, por alguns cuidadores, que as crianças com autismo pareciam demonstrar "mais vontade de brincar", "mais interesse e consciência pelos pares" e estavam "mais atentas" do que antes do início dos jogos (Kok et al., 2002). Esses resultados decorreram muito mais da brincadeira facilitada, uma vez que na dirigida houve maior frequência de brincadeira inapropriada e ausência de iniciações comunicativas.

Yang et al. (2003), em um estudo realizado em Taiwan analisaram os efeitos da participação de duas crianças com autismo, um menino, com 6 anos e 5 meses de idade, e uma menina, com 7 anos e 11 meses, em jogos em grupo, sobre a brincadeira simbólica e social, tanto na escola quanto em casa, com três crianças com DT, na mesma faixa etária. Os encontros ocorreram duas vezes por semana, em sessões de aproximadamente 60 minutos, durante seis meses, totalizando 35 sessões. Para examinar os efeitos da participação nesses grupos sobre a brincadeira social e simbólica foi utilizada uma combinação de metodologia quantitativa e qualitativa. A avaliação dos dados foi realizada por dois codificadores independentes. A média dos níveis de concordância entre os codificadores para a dimensão cognitiva e simbólica foi de $91 \%$, e de $88 \%$ para a dimensão social dos jogos nos dois contextos (casa e escola). Enquanto participaram dos grupos, as crianças com autismo apresentaram notáveis ganhos na interação social recíproca e no jogo simbólico, que envolvia o jogo paralelo e a proximidade com os pares, observação acerca do que as outras crianças faziam e a presença de um foco comum de interesse. Esse resultado demonstra que as crianças com autismo são capazes de brincar de faz-de-conta, ainda que de forma limitada, e de apresentar crescente envolvimento social em brincadeiras com pares, quando há facilitação de adultos, em um setting estruturado. Os pais das crianças com autismo, na entrevista realizada, relataram que, pela primeira vez, elas desenvolveram amizades com outras crianças.

Mesmo nos casos de autismo severo, propostas de intervenção também são oferecidas com o objetivo de promover a interação social dessas crianças. O estudo de Whitaker (2004) investigou as implicações do jogo compartilhado entre 10 crianças com autismo severo, com idade entre 6 e 7 anos, educadas em uma unidade anexa a uma escola comum, e seus pares com DT, que receberam treinamento sobre algumas técnicas de interação. As crianças se encontraram em sessões semanais, totalizando 20 semanas, destinadas a promover o jogo compartilhado e a comunicação. Jogo compartilhado foi definido como o tempo gasto com proximidade física com outra criança, atenção no jogo e brincar/jogar com os mesmos materiais durante a codificação de intervalos de gravação de 10 segundos. De acordo com Whitaker (2004), os resultados foram encorajadores. Análises das sessões de jogos revelaram que os pares com DT foram capazes de envolver e manter as crianças com autismo em níveis mais elevados de jogos compartilhados. A frequência de solicitação das crianças com autismo também aumentou substancialmente ao longo do projeto, apesar dos níveis de iniciação de atenção compartilhada permanecerem consistentemente baixos. Esse resultado é compreensível, uma vez que o comprometimento na atenção compartilhada é um dos principais marcadores, no autismo (Associação Psiquiátrica Americana, 2002)

No estudo de Van Berckelaer-Onnes (2003), por exemplo, 24 crianças com autismo, que participaram de um programa de intervenção individual (Toy-Play), desenvolvido com base em brincadeiras e jogos, foram retestadas um ano após a sua realização. No reteste, 16 crianças apresentaram regressão nos jogos e nas brincadeiras. Segundo o autor, isso pode ter ocorrido pela interrupção dessas atividades com o término da intervenção, uma vez que as oito crianças que não apresentaram regressão (algumas delas inclusive melhoraram) foram atendidas em uma creche em que a estimulação dessas atividades era diária. Diante de tais resultados, o autor propôs um segundo programa de estimulação continuada, seguindo a mesma linha de desenvolvimento do primeiro programa (Toy-Play), mas com maior ênfase no jogo relacional e funcional e, se possível, no jogo simbólico. O objetivo era implementar habilidades recém desenvolvidas no jogo, em situações da vida diária, e 
que envolveria um instrutor e pequenos grupos de crianças que já haviam participado da intervenção anterior. Para o autor, o desenvolvimento desse segundo programa reflete a necessidade de estimular continuamente o jogo de crianças com autismo, especialmente quando este envolve outras crianças, já que para elas o interesse natural para explorar o mundo e as relações encontra-se comprometido.

Ainda sobre intervenções, Bellini, Peters, Benner e Hopf (2007) realizaram uma meta-análise a fim de verificar a efetividade destas sobre as habilidades sociais, na escola, para crianças e adolescentes com autismo. Foram examinados 55 estudos e os resultados se mostraram inconclusivos, uma vez que tais intervenções foram minimamente efetivas para crianças com autismo, por terem sido realizadas em ambientes criados para este fim e, por isso, restritos e descontextualizados, resultando, assim, na dificuldade para manter e generalizar os resultados. Além disso, os autores também verificaram que o cuidado com algumas especificidades na realização de tais intervenções pode auxiliar para que os resultados sejam mais efetivos, tais como: considerar as características específicas dos participantes, ou seja, suas principais dificuldades na interação; o ambiente, o qual deve ser o mais natural possível e não criado apenas para a realização da intervenção; e a descrição dos procedimentos que serão utilizados na intervenção, tais como tipos de atividades desenvolvidas (jogos, brincadeiras), tempo de realização da intervenção, participantes envolvidos, etc.

Por outro enfoque, resultados encorajadores são encontrados nos poucos estudos que têm investigado a interação social de crianças com autismo, com idades entre 3 e 10 anos, em situação de inclusão escolar, cujo foco é o ambiente natural, as situações de brincadeira livre e as interações sociais espontâneas dessas crianças com seus pares com DT (Anderson et al., 2004; Höher-Camargo, 2007; Jahr et al., 2007; Macintosh \& Dissanayake, 2006).

Há alguma evidência de que o ambiente físico e social possa afetar os modelos de interação com pares, o que está de acordo com os resultados encontrados no estudo de Anderson et al. (2004), realizado na Nova Zelândia. Esses pesquisadores verificaram que as interações espontâneas das crianças com autismo com seus pares com DT, com um média de idade de 8 anos, foram mais difíceis quando um adulto permanecia próximo das crianças com autismo, no pátio da escola, por exemplo. Ou seja, parece que os professores acabavam representando uma barreira para que a interação entre crianças com autismo e com DT ocorresse livremente. A possível explicação para isso, segundo os autores, é que como as atividades no pátio não fazem parte do currículo escolar, os professores auxiliares talvez estivessem em dúvida sobre seu papel nesse contexto e, assim, raramente facilitaram ou estimularam brincadeiras ou interações entre pares (Anderson et al., 2004). Além disso, outra explicação plausível, segundo os pesquisadores, é que crianças com DT, a fim de se manterem longe do professor auxiliar, podem evitar interagir com a criança com autismo. A presença constante da "sombra" de um adulto impede que as crianças brinquem mais livremente, uma vez que ele está atento para impedir atividades que possam ser consideradas "perigosas". Esse papel de "vigia" que o adulto assume faz com que as crianças com DT mantenham-se longe dele e, consequentemente, das crianças com autismo, de quem ele está encarregado de cuidar.

Os dados desse estudo sugerem que a prática atual de empregar profissionais de apoio para auxiliar crianças com autismo, na escola comum, não têm promovido a integração social dessas crianças, conforme apontado por Anderson et al. (2004). Resultados semelhantes foram verificados em um estudo brasileiro (Höher-Camargo, 2007), que investigou o padrão de interação social de uma criança pré-escolar com autismo. Esse estudo demonstrou que as atividades realizadas no pátio, onde as crianças podiam brincar livremente, proporcionaram melhor integração da criança com autismo com o grupo, e vice-versa, assim como o desenvolvimento de comportamentos mais adaptativos do que as atividades realizadas em sala de aula. Uma explicação dada pela autora para essa redução da competência social da criança com autismo em sala de aula parece decorrer do caráter das atividades desenvolvidas nesse ambiente. Tais atividades exigiriam maior compreensão da linguagem e do pensamento simbólico (Ex: desenhar, jogos de construção, etc.), aspectos que fazem parte do repertório de dificuldades características do autismo.

Achados semelhantes também são encontrados no estudo de Macintosh e Dissanayake (2006), que investigou as interações livres das crianças em uma escola comum, sem a presença de adultos. Nesse estudo participaram 20 crianças com autismo, 19 crianças com síndrome de Asperger e 17 crianças com DT, emparelhadas na idade cronológica (4 anos a 10 anos e 10 meses) e idade mental geral (QI acima de 70). Nesse contexto, as crianças com DT faziam convites sociais para as crianças com autismo tanto quanto faziam para seus pares típicos, e embora as crianças com autismo ou síndrome de Asperger fossem frequentemente isoladas socialmente, em relação aos seus pares com DT, elas se mostraram capazes de, espontaneamente, engajarem-se socialmente com as outras crianças.

Porém, a verificação desses resultados não significa que as interações das crianças com autismo ocorram da mesma maneira que as interações das crianças com DT. Pelas próprias características do autismo, dificuldades também são observadas e, conforme o esperado, no estudo de Macintosh e Dissanayake (2006), os grupos clínicos (autismo e síndrome de Asperger) foram significativamente menos hábeis do que o grupo com DT para interagir socialmente com seus pares, para manter uma interação social de caráter mais contínuo, ou para estar em interação com três ou mais pares ao mesmo tempo. De qualquer forma, o estudo contesta a noção caricaturizada de uma criança com autismo não-interativa, conforme alguns autores têm enfatizado (ver Bosa, 2002). Um dos aspectos que parece auxiliar as crianças com autismo a estabelecer interações, além da qualidade do ambiente e da facilitação por parte de adultos, é o nível cognitivo e o funcionamento adaptativo/adaptável de cada criança. O estudo de Jahr et al. (2007) apresenta evidências para essa afirmação ao investigar a relação desses dois últimos fatores com a habilidade para interagir ou não com seus pares. Os resultados demonstraram que as crianças com autismo de "alto-funcionamento", isto é, sem deficiência intelectual e com um bom funcionamento adaptativo, foram mais envolvidas em sustentar a interação do que aquelas com maior comprometimento cognitivo e menor funcionamento adaptativo. 
Assim, conclui-se que o desenvolvimento da habilidade das crianças com autismo para se engajar em interações com pares depende das características da própria criança, mas, sobretudo, da oportunidade de poder conviver, o mais cedo possível, em espaços que permitam o desenvolvimento dessas interações, como a escola.De fato, Howes e Phillipsen (1998) ressaltam a importância de experiências precoces com pares para o desenvolvimento da competência social das crianças. Esses autores salientam que a habilidade para engajar-se em jogos/brincadeiras complexas nas idades de 1 a 3 anos e em outros períodos pré-escolares está associado a uma maior presença de comportamentos pró-sociais e a uma menor agressividade na idade escolar.

\section{Conclusão}

Conforme o exposto, os resultados dos estudos que utilizaram programas de intervenção baseados no treinamento dos pares mostraram-se efetivos apenas temporariamente, já que os efeitos positivos obtidos não se mantiveram com o término de tais programas. Isso significa que, até o momento, parece haver poucas evidências de que esse tipo de intervenção suscite um processo real de mudança nas crianças com autismo, com habilidades sociais mais duradouras. Por outro lado, as pesquisas que investigam as interações sociais das crianças com autismo, no contexto de inclusão escolar, em ambientes naturais, têm apresentado resultados mais encorajadores a esse respeito, mas estes ainda são escassos e, portanto, longe de serem conclusivos.

Considerando-se os estudos de forma conjunta, observa-se que raramente a questão do contexto das brincadeiras é entrelaçada com outros aspectos do desenvolvimento da criança e do ambiente educativo. Por exemplo, quais as características dessas interações? Em que situações as crianças tendem a responder melhor? Quais as características das brincadeiras que parecem favorecer as interações? Quais as características dos objetos/brinquedos que parecem atrair o interesse da criança? Quais as características da prática do educador que parecem ser mais efetivas para o desenvolvimento social dessas crianças, ao longo do ano? Quais as características das escolas? Como é a formação básica e continuada dos educadores? Como a escola se organizou para receber as crianças com autismo? Como é a relação escola-família? Essas questões parecem constituir um campo investigativo promissor ao apontar para a necessidade de delineamentos de projetos mais amplos, utilizando estudos de casos e metodologias com ênfase nos aspectos qualitativos dos construtos investigados, tanto quanto os quantitativos, e um referencial teórico que contemple o contexto sociocultural no qual os participantes se inserem.

Finalmente, nem sempre os estudos fornecem detalhes sobre a sintomatologia da criança com autismo, nível adaptativo e cognitivo, linguagem, etc., dificultando a compreensão dos resultados. A desconsideração desses aspectos conduz a uma noção equivocada de que essas crianças são "todas iguais", além de, metodologicamente, tornar mais difícil a possibilidade de replicação dos estudos.

\section{Referências}

Almeida, A. (1997). As relações entre pares em idade escolar. Um estudo de avaliação da competência social pelo método Q-sort. Tese de Doutorado, Universidade do Minho, Portugal.

Anderson, A., Moore, D. W., Godfrey, R., \& Fletcher-Flinn, C. M. (2004). Social skills assessment of children with autism in free-play situations. Autism, 8, 369-385.

Associação Psiquiátrica Americana. (2002). Manual diagnóstico e estatístico de transtornos mentais. Porto Alegre: Artes Médicas.

Baron-Cohen, S., \& Bolton, P. (1993). Autism: The facts. New York: Oxford University Press.

Batista, M. W., \& Enumo, S. R. (2004). Inclusão escolar e deficiência mental: Análise da interação social entre companheiros. Estudos de Psicologia, 9, 101-111.

Baptista, C. R. (2006). Educação especial e o medo do outro: “Attento al segnalati!”. In C. R. Baptista (Ed.), Inclusão e escolarização: Múltiplas perspectivas (pp. 17-29). Porto Alegre: Mediação.

Bellini, S., Peters, J. K., Benner, L., \& Hopf, A. (2007). A metaanalysis of school-based social skills interventions for children with autism spectrum disorders. Remedial \& Special Education, 28, 153-162.

Biaggio, A. M. B. (1976). Psicologia do desenvolvimento. Petrópolis: Vozes.

Bosa, C. (2002). Autismo: Atuais interpretações para antigas observações. In C. R. Baptista \& C. Bosa (Eds.), Autismo e educação: reflexões e propostas de intervenção (pp. 21-39). Porto Alegre: Artmed.

Bruner, J. (1997). Atos de significação. Porto Alegre: Artes Médicas.

Del Prette, Z. A. P., \& Del Prette, A. (2005). Psicologia das habilidades sociais na infância: Terapia e educação. Petrópolis: Vozes.

Fuller, R., \& Jill, E. (2006). Age-related changes in the social inclusion of children with autism in general education classrooms. Dissertation Abstracts International Section A: Humanities and Social Sciences, 66(7-A), 2493.

Gonzalez-Lopez, A., \& Kamps, D. M. (1997). Social skills training to increase social interactions between children with autism and their typical peers. Focus on Autism and Other Developmental Disabilities, 12, 2-14.

Hartup, W. W. (1989). Social relationships and their developmental significance. American Psychologist, 44, 120-126.

Höher-Camargo, S. (2007). Interação social com pares, inclusão escolar e autismo: Um estudo de caso comparativo. Dissertação de Mestrado, Universidade Federal do Rio Grande do Sul, Porto Alegre.

Howes, C., \& Phillipsen, L. (1998). Continuity in children's relations with peers. Social Development, 7, 340-349.

Jahr, E., Eikeseth, S., Eldevik, S., \& Aase, H. (2007). Frequency and latency of social interaction in an inclusive kindergarten setting: A comparison between typical children and children with autism. Autism, 11, 349-363.

Kamps, D. M., Leonard, B. R., Vernon, S., Dugan, E. P., Delquadri, J. C., Gershon, B., ... Folk, L. (1992). Teaching social skills to students with autism to increase peer interactions in an integrated first-grade classroom. Journal of Applied Behavior Analysis, 25, 281-288.

Klin, A. (2006). Autismo e síndrome de Asperger: Uma visão geral. Revista Brasileira de Psiquiatria, 28, 3-11. 
Kok, A. J., Kong, T., \& Bernard-Opitz, V. (2002). A comparison of the effects of structured play and facilitated play approaches on preschoolers with autism: A case study. Autism, 6, 181-196.

LeGoff, D. B. (2004). Use the Lego as a therapeutic medium for improving social competence. Journal of Autism and Developmental Disorders, 34, 557-571.

Macintosh, K., \& Dissanayake, C. L. (2006). A comparative study of the spontaneous social interactions of children with highfunctioning autism and children with Asperger's disorder. Autism, 10, 199-220.

McConnell, S. R. (2002). Interventions to facilitate social interaction for young children with autism: Review of available research and recommendations for educational intervention and future research. Journal of Autism and Developmental Disorders, 32, 351-372.

Nelson, C., Nelson, A. R., McDonnell, A. P., Johnston, S. S., \& Crompton, A. (2007). Keys to play: A strategy to increase the social interactions of young children with autism and their typically developing peers. Education and Training in Developmental Disabilities, 42, 165-181.

Pizzinato, A., \& Sarriera, J. C. (2003). Competência social infantil: Análise discriminante entre crianças imigrantes e não imigrantes no contexto escolar de Porto Alegre. Psicologia em Estudo, 8, 115-122.

Premack, D., \& Woodruff, G. (1978). Does the chimpanzee have a "theory of mind"? Behavioural and Brain Sciences, 4, 515-526.

Rogers, S. (2000). Interventions that facilitate socialization in children with autism. Journal of Autism and Developmental Disorders, 30, 399-409.
Sanini, C., Ferreira, G. D., Souza, T. S., \& Bosa, C. A. (2008). Comportamentos indicativos de apego em crianças com autismo. Psicologia: Reflexão e Crítica, 21, 60-65.

Silveira, F., \& Neves, M. (2006). Inclusão escolar de crianças com deficiência múltipla: Concepções de pais e professores. Psicologia: Teoria e Pesquisa, 22, 79-86.

Terpstra, J.E., \& Tamura, R. (2007). Effective social interaction strategies for inclusive. Settings. Early Childhood Education Journal. 35(5), 405-411.

Van Berckelaer-Onnes, I. A. (2003). Promoting early play. Autism, 7, 415-423.

Waters, E., \& Sroufe, L. A. (1983). Social competence as a developmental construct. Developmental Review, 379-97.

Whitaker, P. (2004). Fostering communication and shared play between mainstream peers and children with autism: Approaches, outcomes and experiences. British Journal of Special Education, 31, 215-222.

Yang, T. R., Wolfberg, P. J., Wu, S. C., \& Hwu, P. Y. (2003). Supporting children on the autism spectrum in peer play at home and school: Piloting the integrated play groups model in Taiwan. Autism, 7, 437-453.

Recebido em 10.01.2011

Primeira decisão editorial em 21.06.2011

Versão final em 28.08.2011

Aceito em 06.09.2011 